\title{
Assessment of Depressive Symptoms in Deaf Persons
}

\author{
Philip Zazove, MD, Helen E. Meador, PhD, James E. Aikens, PhD, \\ Donald E. Nease, MD, and Daniel W. Gorenflo, PhD
}

Background: Evidence suggests that Deaf people may have a greater prevalence of depressive symptoms. However, it is unclear whether commonly used written screening instruments are accurate with this population because of their unique cultural and linguistic factors.

Setting: Deaf persons $(n=71)$ residing in southeastern Michigan.

Methods: Subjects completed the Zung Self-Rated Depression Scale in both the written (ZSDS-W) and American Sign Language (ZSDS-S) formats and the Hamilton Depression Scale (HAM-D) in American Sign Language in counterbalanced order, followed by a Test of Reading Comprehension (TORC). They also provided extensive data on demographic, hearing loss, language use, social and health care use variables.

Results: Mean subject age was 52 years, $63 \%$ of subjects were women, $95 \%$ were Caucasian, and $47 \%$ were married (87\% to another deaf/hard of hearing person). Thirty percent had a college degree and $20 \%$ had less than a 10th grade education. The ZSDS-W and ZSDS-S scores were highly correlated $(r=$ $+0.79)$, although the mean ZSDS-W score was 2.8 points higher $(P=.001)$. The ZSDS-S correlated more highly $(r=0.80)$ with the HAM-D than the ZSDS-W $(r=0.71)$. There was a significant interaction effect $(P<.001)$ such that the ZSDS-W and HAM-D were significantly associated among higher literacy subjects $(\beta=0.80, P<.001)$ but not lower literacy subjects $(\beta=0.20, P=.183)$. There were no other significant associations between depression scores and numerous demographic, educational, hearing loss, social or language variables.

Conclusions: Compared with the conventional ZSDS-W, the ZSDS-S more accurately assesses depression severity among deaf persons with lower English literacy. However, given the greater sensitivity of the ZSDS-W and the practical barriers to ZSDS-S in physician practices, further research should determine which modality is best for routine depression screening. (J Am Board Fam Med 2006;19:141-7.)

Approximately $10 \%$ of Americans have a hearing loss (30 million people), of whom 10\% have a severe to total loss. ${ }^{1,2}$ Approximately 1 million Americans use American Sign language (ASL) as their main means of communication. Hearing loss is the second most common disability in America, following the broad category of orthopedic deformities. ${ }^{3}$ Primary care physicians see $d /$ Deaf and hard of hearing $(\mathrm{D} \& \mathrm{HH})$ patients regularly; our

Submitted 24 May 2005; revised 19 August 2005; accepted 6 September 2005.

From the University of Michigan Health System, Department of Family Medicine, Ann Arbor, MI.

Funding: This research was funded by the Blue Cross Blue Shield Foundation of Michigan and National Institute on Deafness and Other Communication Disorders (NIDCD) Grant PIRAP 556.H.

Conflict of interest: none declared.

Corresponding author: Philip Zazove, MD, 2301 Commonwealth Blvd., Ann Arbor, MI 48105-2945 (E-mail: pzaz@umich.edu). prior work confirms the above prevalence of hearing loss in primary care patients. ${ }^{4}$

The capital D "Deaf" refers to persons who communicate primarily via American Sign Language (ASL), as opposed to lower case d "deaf," which refers to persons who communicate primarily via spoken language (albeit often with difficulty). The use of the term d/Deaf means both groups are being referred to.

D\&HH persons may have an elevated rate of depressive symptoms, according to research on Deaf college students, older D\&HH persons and Deaf residential school students. ${ }^{5-9}$ Much of this work was based on the Beck Depression Inventory-II (BDI-II), which has been validated for assessing Deaf college students. ${ }^{6}$ However, assessing depressive symptoms in the general D\&HH population is complicated by linguistic and literacy issues. Only $13 \%$ of $\mathrm{D} \& \mathrm{HH}$ persons ever attended college (versus approximately $30 \%$ of hearing persons), ${ }^{10}$ and the mean reading level among people 
who lose their hearing at a young age is at the 4th to 6 th grade level. ${ }^{11}$ Moreover, D\&HH persons are a linguistically diverse group. Deaf persons often have great difficulty communicating in English because ASL has a unique grammar, syntax, and set of idioms. ${ }^{12}$ Therefore, even well-educated Deaf persons may not understand simple written medical terms such as "nausea" and "allergic,"13 and as a group have greater difficulty interacting with health care professionals than other non-English speaking minorities. ${ }^{14}$

Written self-report is obviously the easiest method for assessing depressive symptoms in the clinical setting. However, the above-noted variations in language and literacy raise the question of whether common written depression scales are appropriate for use with the general Deaf population. Although video-based instruments are a potential solution, these remain relatively impractical for the average medical office. ${ }^{6,15}$ To examine the potential validity of such scales for Deaf persons, we evaluated signed versus written forms of a common instrument, the Zung Self-Rated Depression Scale (ZSDS), ${ }^{16}$ along with a Hamilton Depression Rating Scale (HAM-D) ${ }^{17}$ interview conducted in ASL. We chose the ZSDS rather than the BDI-II, which is the only instrument that has been shown to be accurate in Deaf persons. Others, such as the BDI-I for example, are not acceptable. The ZSDS is not only highly correlated with the BDI-II, ${ }^{18}$ it is also easier to translate to ASL. The BDI-II requires respondents to simultaneously consider 4 complex possible responses that differ for each item. In contrast, the ZSDS response format is constant across items, making it easier to translate. By using the Zung (which is correlated with the BDI-II), rather than other instruments that are shorter, such as the PHQ-9 scale, we reduced the likelihood of introducing instrument bias. Finally we examined whether the validity of written depression assessment varies by English-reading literacy using the Test of Reading Comprehension Subtest 2-Syntactic Similarities (TORC-SS). ${ }^{19}$

\section{Methods}

Study subjects were solicited from participants in our multimedia study for Deaf persons ${ }^{20}$ as well as through our extensive longstanding contacts with Michigan's D\&HH population. All persons age 18 and over who agreed to participate completed the
Gallaudet Hearing Loss Scale (as used in the $\mathrm{Na}$ tional Health Interview survey) $)^{2}$ to verify they had a severe or profound hearing loss. They next completed a consent form, then viewed a video-based computer program to assess knowledge of cancer screening and prevention (for the multimedia study), and provided information about their demographics, hearing loss variables (age of onset of loss, cause of loss, etc), family hearing loss history, language history (current language preference, language used in school, language used at home, etc), health care utilization history, presence of health problems, and the source of their health care information among other items. Mean questionnaire completion time was 40 minutes.

Depression study participants were enrolled individually at a separate time and place from the multimedia study, at which time they completed only the depression study protocol. These people completed a consent form followed by the questionnaire portion of the multimedia study before participating in the depression study. They then were given the written Zung Self-Rating Depression Scale (ZSDS-W), ASL version of the ZSDS (ZSDS-S), the HAM-D in the subject's preferred language (usually ASL), and the TORC-SS. The TORC-SS was administered in written English format. The HAM-D, which measures the severity of depressive symptoms in patients, was used as our "standard," ie, it was the instrument used to assess the accuracy of the results of each of the 2 ZSDS tests. Because it involves follow-up questions and probing of answers to accurately score each of 17 areas, it is most accurate when used in the respondent's preferred language. The Syntactic Similarities Subtest 2 of the TORC was selected (rather than the Sentence Sequencing or Paragraph Reading section) because it tests the comprehension of syntactic structures regardless of sentence sequence, ie, forcing the reader to choose between 2 similar sentences that have different syntax (eg, "the picture that was drawn by the girl is finished," vs. "the girl has finished drawing her picture."). This relates to the ZSDS- $W$, which involves reading a variety of sentences with different syntax and that do not follow each other sequentially. Administration order was systematically counterbalanced such that each instrument was given first, second, and third the same number of times; the TORC-SS was always administered last. To verify translation accuracy, a certified ASL interpreter was present 


\begin{tabular}{|c|c|c|}
\hline & Percentage or Mean \pm SD & Median (and/or range) \\
\hline Age in years & $52 \pm 16$ & $(20-89)$ \\
\hline Gender (female) & $63 \%$ & \\
\hline Ethnic/racial minority & $5 \%$ & \\
\hline $\begin{array}{l}\text { Marital status } \\
\text { Married } \\
\text { D\&HH spouse }\end{array}$ & $\begin{array}{l}47 \% \\
87 \%\end{array}$ & \\
\hline $\begin{array}{l}\text { Education } \\
\quad<10 \text { th grade } \\
\text { College degree }\end{array}$ & $\begin{array}{l}20 \% \\
30 \%\end{array}$ & \\
\hline $\begin{array}{l}\text { Employment status } \\
\text { Employed } \\
\text { Full-time }\end{array}$ & $\begin{array}{l}50 \% \\
83 \%\end{array}$ & \\
\hline Household income (mean) & $\$ 25,000$ & $(\$ 5,000-\$ 80,000)$ \\
\hline $\begin{array}{l}\text { Age of Onset of Hearing Loss } \\
\quad<1 \text { year } \\
\geq 1 \text { year }\end{array}$ & $\begin{array}{l}50 \% \\
50 \%\end{array}$ & \\
\hline Member of the Deaf community & $76 \%$ & \\
\hline $\begin{array}{l}\text { Language use (multiple answers w } \\
\text { At home } \\
\text { ASL } \\
\text { Signed contact language } \\
\text { Signed English } \\
\text { Written English } \\
\text { English speech only }\end{array}$ & $\begin{array}{l}70 \% \\
31 \% \\
17 \% \\
14 \% \\
30 \%\end{array}$ & \\
\hline $\begin{array}{l}\text { With people who are D\&HH } \\
\text { ASL } \\
\text { Signed contact language } \\
\text { Signed English }\end{array}$ & $\begin{array}{l}74 \% \\
22 \% \\
21 \%\end{array}$ & \\
\hline $\begin{array}{l}\text { With people who are hearing } \\
\text { Spoken English } \\
\text { Written English } \\
\text { English with interpreter }\end{array}$ & $\begin{array}{l}36 \% \\
33 \% \\
28 \%\end{array}$ & \\
\hline
\end{tabular}

D\&HH, Deaf and hard of hearing; ASL, American Sign Language.

during practice back-translations of the ZSDS-S and HAM-D by the interpreter involved in our study, and agreement was reached on how to present each question of the ZSDS and HAM-D. This verified that these instruments were being appropriately translated.

The ZSDS scores were obtained using the guidelines provided by the company. For each question, a score from 1 to 4 was given, depending on the specific answer. These were then totaled to give a final total score. Likewise, the HAM-D had a score from 0 to 2 for some questions, from 0 to 3 for one question, and from 0 to 4 for some questions. These were also totaled to give a final total score. The TORC-SS had a score from 0 to 20 , which was converted to a reading level according to the TORC manual.

The study protocol was approved by the University of Michigan Medical School Institutional Review Board. Data were analyzed using a combination of descriptive statistics, Pearson correlation,
Student's $t$ test, and OLS multiple regression analysis, after skewed distributions were rank-transformed.

\section{Results}

\section{Sample Characteristics}

Seventy-one subjects participated in the study, 50\% of whom were also enrolled in the multimedia study. The mean age was 52 years $(\mathrm{SD}= \pm 16$, range 20 to 89 ), $63 \%$ were female, $95 \%$ were Caucasian, and $47 \%$ were married $(87 \%$ to another D\&HH person). Thirty percent had a college degree and 20\% had less than a 10th grade education. A summary of the demographic data is presented in Table 1, as are detailed findings on hearing loss, languages used to communicate, and other relevant variables. Both Deaf and deaf persons were involved, although most were Deaf as evidenced by $76 \%$ agreeing with the statement they were part of the Deaf community. 


\begin{tabular}{lccc}
\hline Measure & Mode of Administration & Mean \pm SD & Range \\
\hline Zung Self-Rating Depression Scale (ZSDS-W) & Written & $39.3 \pm 10.2$ & $20-59$ \\
Zung Self-Rating Depression Scale (ZSDS-S) & Signed & $36.5 \pm 10.8$ & $20-60$ \\
Hamilton Depression Rating Scale (HAM-D) & Signed & $9.3 \pm 5.9$ & $0-21$ \\
Test of Reading Comprehension (TORC) & Written & $6.8 \pm 3.4$ & $2.2-16.0$ \\
\hline
\end{tabular}

\section{Descriptive Data}

Table 2 contains descriptive statistics for the 3 depressive symptom measures and the TORC-SS. Responses were distributed across the range of 20 to 60 for both the ZSDS-W and ZSDS-S, whereas the highest HAM-D score (21 in the "severe" range) was virtually at the highest possible score of 23. The TORC-SS scores were also well dispersed; the mean score of 6.8 correlates to almost a 7 th grade reading level.

\section{Correlates of Depression Severity}

None of the 3 depression measures was significantly associated with reading comprehension, use of any type of language at either home or with hearing persons, age, or household income variables (all $r$ values $\leq .22$ ). Nor was there any association of the depression measures with parental hearing status or presence of various medical conditions (including a history of depression). However, all 3 depression measures varied inversely with level of education $(r$ values from -.26 to -.29 ; all $P$ values $<.04$ ).

\section{Associations between ZSDS Scores}

The 2 ZSDS measures correlated $+.79(P<.001)$, and this association was unaffected by adjustments for literacy, comfort with English, language used at home, education, and other potential confounders. Cronbach's $\alpha$ was .85 for both ZSDS versions. The ZSDS-W scores were almost 3 points higher than ZSDS-S scores (mean difference $=2.8, t=3.52$, $P=.001$ ), although this is only $5 \%$ of the possible range, and not a clinically significant increment. Administration order had no detectable effect on depression scores.

\section{Associations between ZSDS and HAM-D Scores}

Because the HAM-D (our reference standard) is designed to measure depressive symptoms severity rather than to function as a screening or diagnostic instrument, we evaluated linear associations be- tween the HAM-D and each ZSDS version. Figure 1 depicts a composite scatter-plot illustrating the linear associations between each ZSDS version and HAM-D scores. The ZSDS-S correlated +.80 $(P<.001)$ with the HAM-D, and the ZSDS-W correlated $+.71(P<.001)$ with the HAM-D.

To further compare the 2 associations in Figure 1 , regression models were fitted to evaluate the increments in HAM-D variance accounted for by the ZSDS-S followed by the ZSDS- $W$, respectively, and also vice versa. Although the ZSDS-S explained a significant increment in HAM-D variance after accounting for the ZSDS-W (see Table 3 , analysis 1 ), the ZSDS-W did not explain additional HAM-D variance after accounting for the ZSDS-S (see Table 3, analysis 2). To assess whether level of education, reading literacy, age, or gender were related to the performance of the ZSDS- $W$, the regression analysis was re-conducted with an additional initial block that used a forward selection entry procedure to select variables for inclusion. Education met entry criteria $(\beta=-.28$, $P=.025)$; however, the ZSDS-W effect was un-

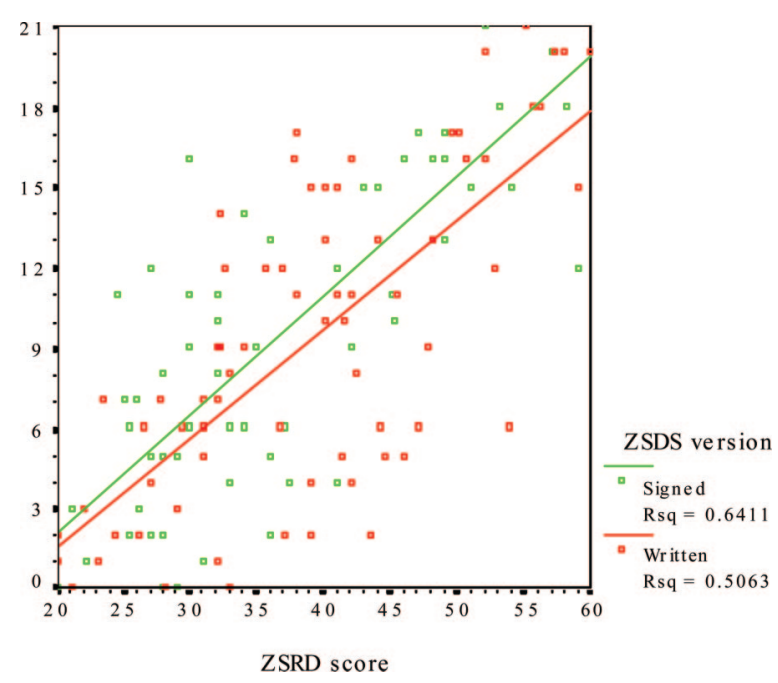

Figure 1. Scatterplot of ZSDS scores versus HAM-D scores. 
Table 3. Results of Regression Analysis of Hamilton Depression Rating Scale (HAM-D) Scores

\begin{tabular}{lcccc}
\hline Analysis & Block & Predictor Variable & Standard $\beta$ & $P(\beta)$ \\
\hline 1 & 1 & ZSDS-W & 0.71 & $<.001$ \\
& 2 & ZSDS-S & 0.64 & $<.001$ \\
2 & 1 & ZSDS-S & 0.80 & $<.001$ \\
& 2 & ZSDS-W & 0.21 & NS \\
\hline
\end{tabular}

ZSDS-W, Zung Self-Rating Depression Scale, written; ZSDS-S, Zung Self-Rating Depression Scale, signed.

changed $(\beta=0.73, P<.001)$, and the education $\mathrm{X}$ ZSDS-W interaction effect did not reach statistical significance $(\beta=0.10, P=.24)$. Although literacy (higher versus lower, as defined by median split on TORC-SS scores) did not have a significant main effect $(\beta=0.09, P=.28)$, there was a significant literacy $\mathrm{X}$ ZSDS-W interaction $(\beta=0.20, P=$ .024; see Figure 2). That is, the ZSDS-W and HAM-D were significantly associated among higher literacy subjects $(\beta=0.80, P<.001)$ but not among lower literacy subjects $(\beta=0.20, P=.183)$. In contrast, literacy did not interact with ZSDS-S scores $(P=.137)$.

\section{Discussion}

There are clearly differences between cultures regarding the acceptance and description of depression. The Deaf culture has traditionally not been very open in acknowledging the disease and people who are affected by it. Moreover, some idioms in English that relate to depression (eg, feeling blue) would not make sense if translated word for word in sign language. We are not aware of any published studies investigating cultural idioms in Deaf or deaf persons. However, the fact that there are differences between English and ASL in how depression can be described highlights the importance of identifying accurate depression screening instruments for the Deaf community, especially if we are to provide them with appropriate treatment.

In this study, we found that although both the signed (ZSDS-S) and written (ZSDS-W) versions of the self-reported Zung Self-Rated Depression Scale correlated well with the standard, the HAM-D interview, the ZSDS-S corresponded significantly better. In particular, this was because the ZSDS-W was less predictive of HAM-D scores for lower literacy than for higher literacy subjects whereas the ZSDS-S was equally predictive across all literacy levels. Both versions performed similarly well among higher literacy subjects, and we did not detect any association between either ZSDS score and other social and demographic factors. Of note, the ZSDS- $W$ yielded significantly higher scores of almost 3 points. As a result, it may have a higher sensitivity to detecting HAM-D-defined probable depression than the ZSDS-S.

In many situations involving Deaf patients—and especially with a large cohort of typical Deaf per-

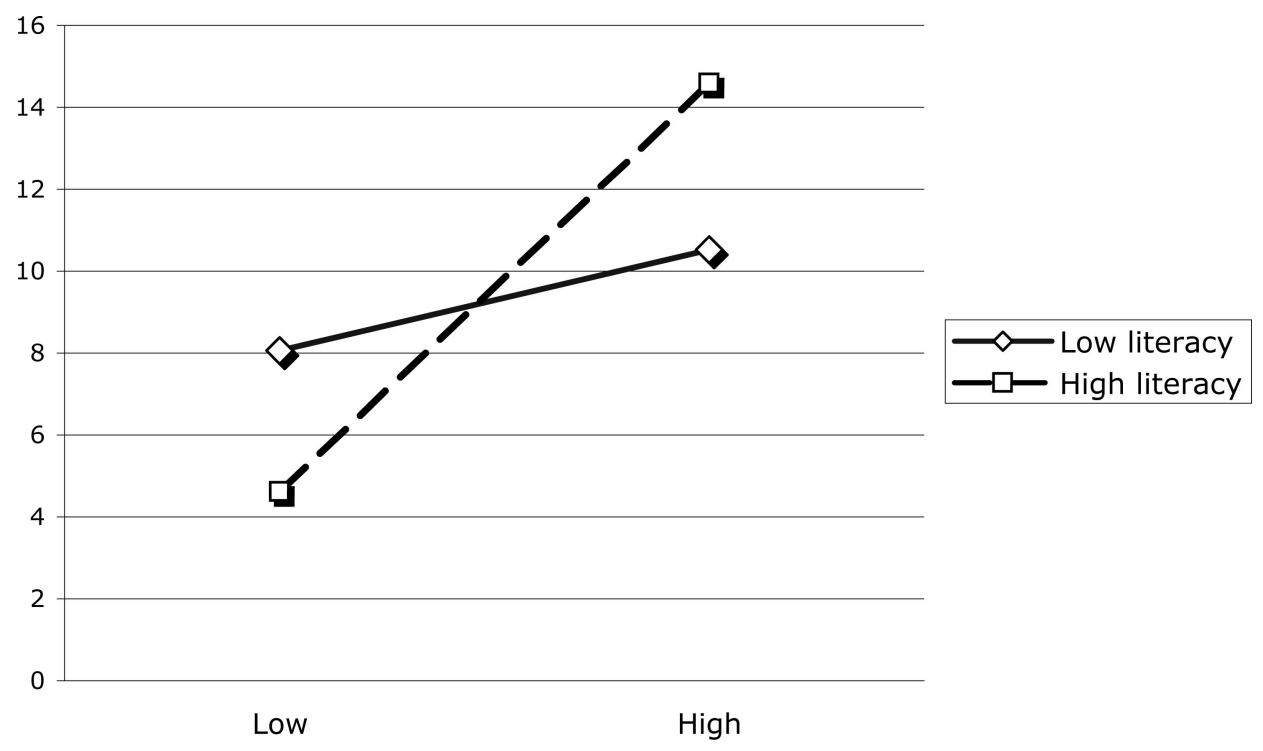

ZSDS-Written score

Figure 2. HAM-D and ZSDS-W scores by literacy level. 
sons who are not good readers-the ZSDS-S is probably the better choice for assessing depressive symptoms. However, few primary care physicians provide ASL translation in their office, and computer or video-based screening instruments remain impractical in most offices. ${ }^{15}$

The Americans with Disabilities Act does mandate that interpreters be used when the patient provides advance notice of the need for them. In such cases, the interpreter can translate the written ZSDS (ie, provide a ZSDS-S) and thus allow the physician to effectively administer the instrument in sign language. However, interpreters may be unavailable up to $50 \%$ of the time, ${ }^{10}$ and their cost can be significant (upwards of $\$ 30 /$ hour with a 2 hour minimum). This reduces the practicality of administering the ZSDS-S to Deaf patients and would be especially the case in rural areas where interpreters are rarely available. ${ }^{10}$

Theoretically, one could administer the ZSDS-W to all d/Deaf patients with higher literacy and use the ZSDS-S only for those with lower literacy. However, there is no feasible way to easily identify literacy levels in patients. The finding of higher scores on the ZSDS-W suggests that it might have superior sensitivity for the presence of depressive disorder, although probably at the expense of lower specificity. If so, it might be the better depression screening instrument for all $d /$ Deaf patients because of its much greater practicality. Patients with elevated ZSDS-W scores would then require subsequent evaluation in their preferred language. Further research is needed to better understand the pros and cons of the ZSDS-W and the ZSDS-S as screening instruments for $\mathrm{d} /$ Deaf patients.

Certain study limitations need to be emphasized. First, the sample was relatively small, and our population was more likely to be educated and Caucasian than the typical d/Deaf population. Sampling bias may also have been introduced by recruiting from a separate study that had aims unrelated to depression. Third, all persons had a severe or profound hearing loss and most were members of the Deaf community. Thus, our results may not generalize to the entire d/Deaf population. For example, oral deaf persons are known to have major communication difficulties of their own as well as lower reading levels, and it is not known whether they need adaptations to written screening instruments. Fourth, we did not compare written and signed ZSDS scores in other languages, and thus our findings may not apply to Deaf populations who use those languages. Finally, our study did not specifically assess syndromal depression. Therefore, we cannot draw conclusions about the presence or absence of clinically significant Major Depressive Disorder.

We conclude that, compared with the ZSDS-W, the ZSDS-S better explains depression severity as assessed by the HAM-D interview for lower literacy d/Deaf persons but the ZSDS-W may have increased sensitivity to detecting depression symptoms. Further research using signed standardized psychiatric interviews can determine which instrument is best for general depression screening in these persons.

\section{References}

1. Adams PF, Hardy AM. Current estimates from the National Health Interview Survey: United States1988. Vital Health Stat 10. 1989;173.

2. Ries PW. Prevalence and characteristics of persons with hearing trouble: United States, 1990-1991. Vital Health Stat 10. 1994;188.

3. Ries PW. Hearing ability of persons by sociodemographic and health characteristics: United States 1982. Vital Health Stat 10. 1982;140.

4. Zazove P, Niemann L, Gorenflo D, et al. The health status and health care utilization of deaf and hardof-hearing persons. Arch Fam Med 1993;2:745-52.

5. Leigh IW, Robins CJ, Welkowitz J, Bond RN. Toward greater understanding of depression in deaf individuals. Am Ann Deaf. 1989;134:249-54.

6. Leigh IW, Anthony-Tolbert S. Reliability of the BDI-II with deaf persons. Rehabil Psychol 2001; 195-202.

7. Magilvy JK. Experiencing hearing loss in later life: a comparison of deaf and hearing-impaired older women. Res Nurs Health 1985;8:347-53.

8. Watt JD, Davis FE. The prevalence of boredom proneness and depression among profoundly deaf residential school adolescents. Am Ann Deaf 1991; 136:409-13.

9. Steinberg AG, Lipton DS, Eckhardt EA, Goldstein M, Sullivan J. The diagnostic interview schedule for deaf patients on interactive video: a preliminary investigation. Am J Psychiatry 1998;1603-4.

10. Michigan Commission on Handicapped Concerns. The hearing-impaired population of Michigan. Lansing (MI): Michigan Department of Labor, Division on Deafness; 1989.

11. Drasgow E. Bilingual/bicultural deaf education: an overview. Sign Language Studies 1993;80:243-66.

12. Moores D. Educating the deaf: psychology, princi- 
ples, and practices. 5th ed. Boston: Houghton Mifflin; 2001.

13. Lass LG, Franklin RR, Bertrand WE, Baker J. Health knowledge, attitudes, and practices of the deaf population in greater New Orleans: a pilot study. Am Ann Deaf. 1978;123:960-7.

14. McEwen E, Anton-Culver H. The medical communication of deaf patients. J Fam Pract 1988;26:28991.

15. Berman BA, Eckhardt EA, Kleiger H, et al. Developing a tobacco survey for deaf youth. Am Ann Deaf. 2000;145:245-55.

16. Zung WW. Development of a rating scale for pri- mary depressive illness. Arch Gen Psychiatry 1965; 13:508-15.

17. Hamilton M. Development of a rating scale for primary depressive illness. Br J Soc Clin Psychiatry 1967;6:278-96.

18. Carroll BJ, Fielding JM, Blashki TG. Depression rating scales, a critical review. Arch Gen Psychiatry 1973;28:361-6.

19. Brown V, Hammill D, Wiederholt JL. Test of reading comprehension. 3rd ed. Austin (TX): PRO-ED, Inc.; 1995.

20. Zazove P, Meador HE, Derry HA, Gorenflow DW, Burdick SW, Saunders EW. Deaf persons and computer use. Am Ann Deaf 2004;148:376-84. 